$\operatorname{der}(1 ; 7)(q 10 ; p 10)$. We observed that MDS patients with $\operatorname{der}(1 ; 7)$ (q10;p10) present male predominance and have a better outcome than the $-7 / \operatorname{del}(7 q)$ group (Supplementary Table 4). Our findings revealed that the mutation spectrum of patients with $\operatorname{der}(1 ; 7)(q 10$; p10) was different from that of MDS with $-7 /$ del(7q). We demonstrate for the first time, to our knowledge, that der $(1 ; 7)$ $(q 10 ; p 10)$ is associated with a high frequency of mutations in RUNX1. Further studies are needed to clarify whether and how mutations of $R U N X 1$ contribute to the pathogenesis of this subtype.

\section{CONFLICT OF INTEREST}

The authors declare no conflict of interest.

\section{ACKNOWLEDGEMENTS}

This work was supported by grants from the Priority Academic Program Development of Jiangsu Higher Education Institutions, the Natural Science Foundation of China (81570139, 81370626, 81070416, 81300424, 81400112), the national key research and development program, 2016YFC0902800), the Innovation Capability Development Project of Jiangsu Province (BM2015004), and Jiangsu Province Natural Science Fund (BE2015639).

\section{AUTHOR CONTRIBUTIONS}

SC and AS were the principal investigators. TZ, YX, JP, QW, LW and DP performed most of the experiments. HL performed structural model of RUNX1. $\mathrm{SC}$ and AS wrote the manuscript.

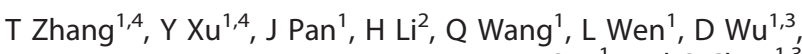
A Sun ${ }^{1}$ and $S$ Chen ${ }^{1,3}$

${ }^{1}$ Jiangsu Institute of Hematology, Key Laboratory of Thrombosis and Hemostasis of Ministry of Health, the First Affiliated Hospital of Soochow University, Suzhou, China;

${ }^{2}$ Department of Molecular Medicine, Beckman Research Institute, City of Hope National Medical Center, Duarte, CA, USA and

${ }^{3}$ Institute of Blood and Marrow Transplantation, Collaborative Innovation Center of Hematology, Soochow University,

Suzhou, China

E-mail: sunaining@suda.edu.cn or chensuning@suda.edu.cn

${ }^{4}$ These authors contributed equally to this work.

\section{REFERENCES}

1 Swerdlow SH, Campo E, Harris NL, Jaffe ES, Pileri SA, Stein H et al. (eds) WHO Classification of Tumours of Haematopoietic and Lymphoid Tissues. IARC Press: Lyon, France, 2008.

2 Haase D, Germing U, Schanz J, Pfeilstöcker M, Nösslinger T, Hildebrandt B et al. New insights into the prognostic impact of the karyotype in MDS and correlation with subtypes: evidence from a core dataset of 2124 patients. Blood 2007; 110: 4385-4395.

3 Schanz J, Steidl C, Fonatsch C, Pfeilstöcker M, Nösslinger T, Tuechler H et al. Coalesced multicentric analysis of 2,351 patients with myelodysplastic syndromes indicates an underestimation of poor-risk cytogenetics of myelodysplastic syndromes in the international prognostic scoring system. J Clin Oncol 2011; 29: 1963-1970.

4 Greenberg PL, Tuechler H, Schanz J, Sanz G, Garcia-Manero G, Solé F et al. Revised international prognostic scoring system for myelodysplastic syndromes. Blood 2012; 120: 2454-2465.

5 Wang L, Ogawa S, Hangaishi A, Qiao Y, Hosoya N, Nanya $Y$ et al. Molecular characterization of the recurrent unbalanced translocation $\operatorname{der}(1 ; 7)(q 10 ; p 10)$. Blood 2003; 102: 2597-2604.

6 Geraedts JPM, Ottolander GJD, Ploem JE, Muntinghe OG. An identical translocation between chromosome 1 and 7 in three patients with myelofibrosis and myeloid metaplasia. Br J Haematol 1980; 44: 569-575.

7 Westman MK, Pedersen-Bjergaard J, Andersen MT, Andersen MK. IDH1 and IDH2 mutations in therapy-related myelodysplastic syndrome and acute myeloid leukemia are associated with a normal karyotype and with $\operatorname{der}(1 ; 7)(q 10 ; p 10)$. Leukemia 2013; 27: 957-959.

8 So CC, Ma ES, Wan TS, Yip SF, Chan LC. Clinicopathological features of unbalanced translocation Der(1;7)(q10;p10) in myeloid neoplasms. Leuk Res 2008; 32: 1000-1001.

9 Sanada M, Uike N, Ohyashiki K, Ozawa K, Lili W, Hangaishi A et al. Unbalanced translocation $\operatorname{der}(1 ; 7)(q 10 ; p 10)$ defines a unique clinicopathological subgroup of myeloid neoplasms. Leukemia 2007; 21: 992-997.

10 Hussain FT, Nguyen EP, Raza S, Knudson R, Pardanani A, Hanson CA et al. Sole abnormalities of chromosome 7 in myeloid malignancies: spectrum, histopathologic correlates, and prognostic implications. Am J Hematol 2012; 87: 684-686.

11 Slovak ML, O'Donnell M, Smith DD, Gaal K. Does MDS with der(1;7)(q10;p10) constitute a distinct risk group? A retrospective single institutional analysis of clinical/pathologic features compared to -7/del(7q) MDS. Cancer Genet Cytogenet 2009; 193: 78-85.

12 Gaidzik VI, Teleanu V, Papaemmanuil E, Weber D, Paschka P, Hahn J et al. RUNX1 mutations in acute myeloid leukemia are associated with distinct clinicopathologic and genetic features. Leukemia 2016; 30: 2160-2168.

13 Haferlach T, Nagata Y, Grossmann V, Okuno Y, Bacher U, Nagae G et al. Landscape of genetic lesions in 944 patients with myelodysplastic syndromes. Leukemia 2014; 28: 241-247.

14 Christiansen DH, Andersen MK, Pedersen-Bjergaard J. Mutations of AML1 are common in therapy-related myelodysplasia following therapy with alkylating agents and are significantly associated with deletion or loss of chromosome arm $7 q$ and with subsequent leukemic transformation. Blood 2004; 104: 1474-1481.

\title{
B7-H4 enhances the differentiation of murine leukemia- initiating cells via the PTEN/AKT/RCOR2/RUNX1 pathways
}

Leukemia (2017) 31, 2260-2264; doi:10.1038/leu.2017.232

Leukemia-initiating cells (LICS) are believed to be responsible for the initiation, development and relapse of leukemia. ${ }^{1}$ To effectively eliminate LICs, new molecular targets and therapeutic strategies for targeting either extracellular or intracellular signaling are needed. Studies from our and other groups have suggested that targeting specific surface immune molecules of LICs, including LILRB2, ${ }^{2} \mathrm{CD} 123,{ }^{3} \mathrm{CD} 47^{4}$ and $\mathrm{CD} 93,{ }^{5}$ may be a promising strategy to block leukemogenesis. To identify more such surface immune molecules, we screened approximately 30 cell surface proteins expressed in immune systems, and found that several immune molecules, including IREM-1, BTLA, CD244, JAM3, B7-H1 and $\mathrm{B} 7-\mathrm{H} 4$, were highly expressed on MLL-AF9-induced human acute myeloid leukemia (AML) cells. ${ }^{6}$ Interestingly, one of the identified candidates $\mathrm{B} 7-\mathrm{H} 4$ was also expressed on one fraction of human LIC-enriched $\mathrm{CD} 34^{+} \mathrm{AML}$ cells as determined by flow cytometric analysis (Supplementary Figure 1a). More importantly, in silico analysis using data extracted from the curated database 
a

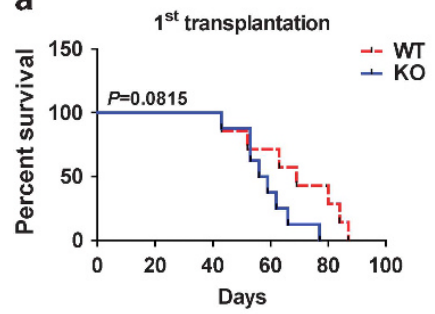

b
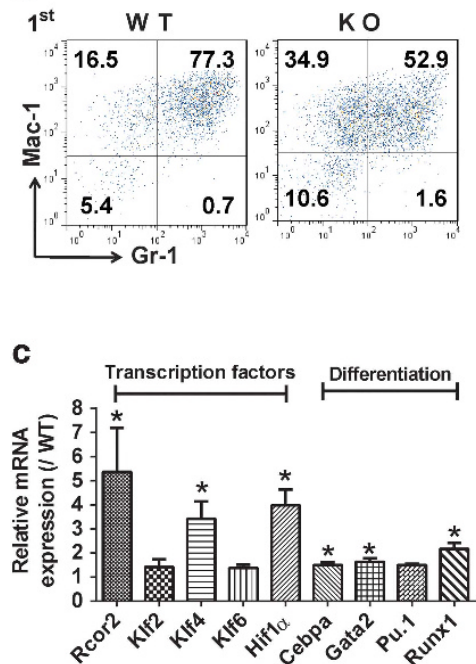
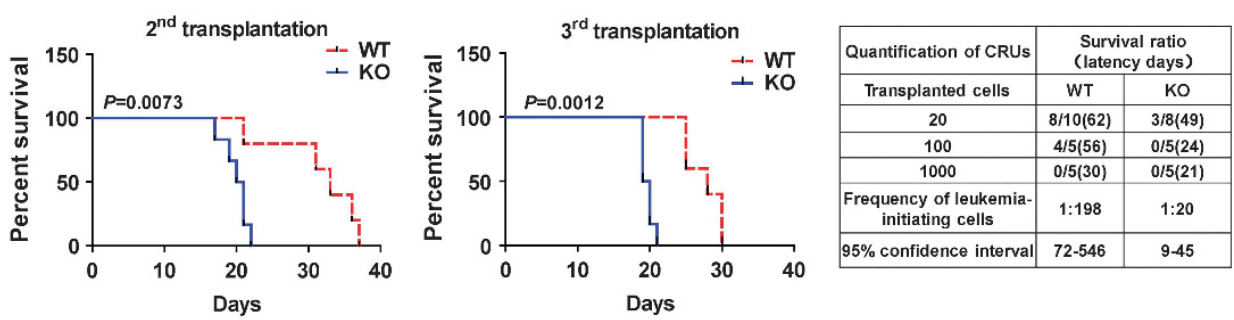
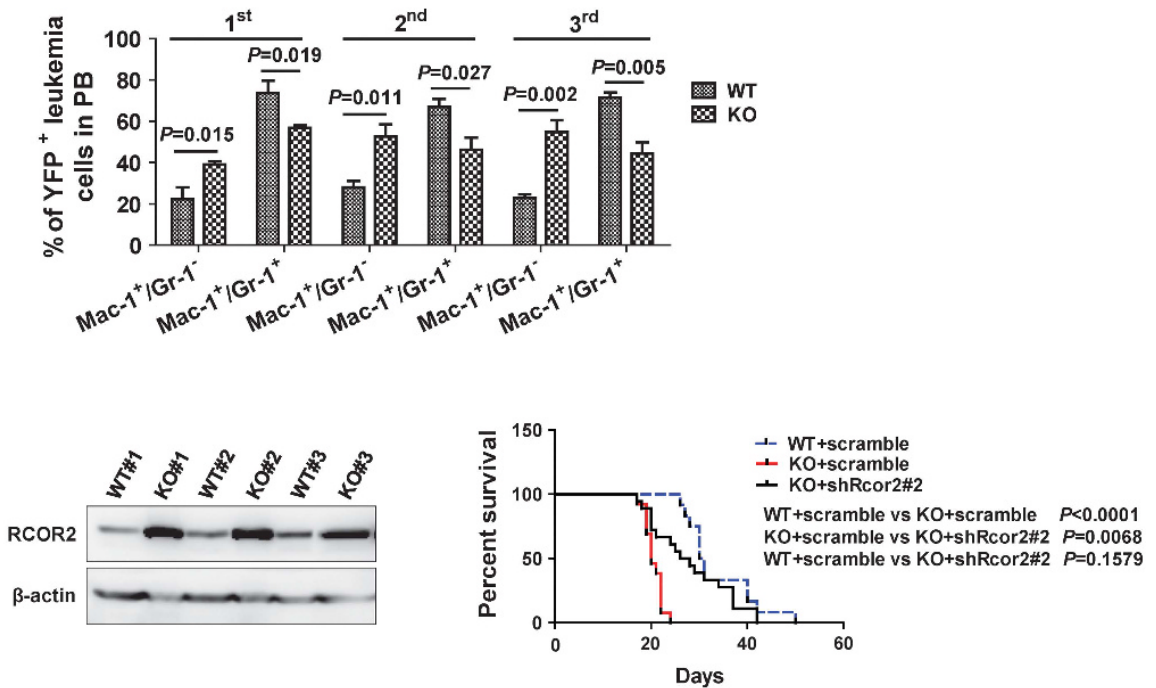

Figure 1. B7-H4 promotes the differentiation of LICs to inhibit leukemogenesis by downregulating the RCOR2 level. (a) Survival was compared between the recipients transplanted with MLL-AF9-infected WT and B7-H4-null (KO) hematopoietic stem/progenitors upon primary transplantation (first, $n=7-8$, log-rank test, first panel), and mice receiving $10000 \mathrm{WT}$ or B7-H4-null YFP ${ }^{+}$door leukemia cells from the primary (second, second panel) and secondary (third, third panel) recipients ( $n=5-6$, log-rank test). Limiting dilution assays comparing the frequencies of LICs in WT and B7-H4-null BM cells. The indicated numbers of YFP ${ }^{+}$leukemia cells isolated from secondary recipients were co-injected with $2 \times 10^{5}$ competitor cells into lethally irradiated recipients (fourth panel). The competitive repopulating units (CRUs) were evaluated using L-Calc software and indicated. (b) Representative flow cytometric analysis of the YFP Mac- $1^{+} \mathrm{Gr}-1^{+}$and $\mathrm{YFP}{ }^{+} \mathrm{Mac}-1^{+} \mathrm{Gr}-1^{-}$leukemia cells in the peripheral blood of primary recipients receiving transplants of MLL-AF9-transduced WT or B7-H4-null hematopoietic stem/progenitors (first panel), and qualification of frequencies of $\mathrm{YFP}{ }^{+} \mathrm{Mac}-1^{+} \mathrm{Gr}-1^{+}$and $\mathrm{YFP} \mathrm{Mac}^{+} 1^{+} \mathrm{Gr}-1^{-}$leukemia cells in the peripheral blood (PB) of primary, secondary and tertiary recipients are shown ( $n=5$, second panel). (c) Potential candidate genes related to transcription factors (important for stemness) and myeloid differentiation were validated in fluorescence activated cell sorting-purified WT or B7-H4-null YFP ${ }^{+}$Mac- $1^{+} \mathrm{c}-\mathrm{Kit}^{+} \mathrm{LICs}^{-}$ using quantitative Reverse transcription-polymerase chain reaction analysis ( $n=3$, first panel), and protein levels of RCOR2 in WT and B7-H4null BM AML cells were measured using immunoblotting ( $n=3$, second panel). Rcor2 was silenced in B7-H4-null AML cells using shRcor2\#2, which were transplanted into the recipient mice. The survival was analyzed among the mice receiving WT cells, B7-H4-null cells and Rcor2knockdown B7-H4-null cells ( $n=12-18$, log-rank test, third panel). mRNA, messenger RNA.

Leukemia Gene Atlas (http://www.leukemia-gene-atlas.org) also showed that B7-H4 expression level was positively correlated with the overall survival of AML patients (Supplementary Figure 1b), indicating that $\mathrm{B} 7-\mathrm{H} 4$ may serve as a tumor suppressor during leukemia development.

B7-H4 is initially found to be expressed on the surfaces of antigenpresenting cells and negatively regulates the activities of $T$ cells or neutrophils by binding to an unknown receptor. ${ }^{7}$ However, the role of B7-H4 in cancer development remains controversial since B7- 44 has been shown to either enhance ${ }^{8}$ or inhibit tumorigenesis ${ }^{9}$ through the evasion of immune surveillance or other unknown mechanisms. To determine the function of B7-H4 in LICs, we used B7-H4 knockout (KO) mice to establish an MLL-AF9-induced AML model and evaluated its role in the development of AML.

As expected, the B7-H4 expression level was only detected on the wild-type (WT), but not B7-H4-null leukemia cells (Supplementary Figure 1c). Interestingly, the frequencies of B7$\mathrm{H}_{4}$-null $\mathrm{YFP}^{+}$leukemia cells in the peripheral blood are slightly higher than those in WT controls upon primary transplantation although there was no significant difference (Supplementary Figures $1 \mathrm{~d}$ and e). We also observed a minor reduction in the survival of the primary B7-H4-null recipients (57.5 vs 69 days, Figure 1a, first panel), indicating that B7-H4 may suppress leukemogenesis. Strikingly, there were much higher percentages of B7-H4-null YFP ${ }^{+}$donor cells in the peripheral blood in the second and third transplantation (Supplementary Figures 1d and e), and survival was also markedly decreased in recipients receiving B7-H4-null donor cells upon the subsequent second and third transplantation compared to those of WT controls (20.5 vs $33,19.5$ vs 28 days, respectively, Figure $1 \mathrm{a}$, second to third panel). More importantly, limiting dilution assays with $\mathrm{YFP}^{+}$ leukemia cells isolated from the second transplant revealed that the LIC frequency was 1 in 20 of B7-H4-null leukemia cells, which was $~ 10$-fold higher than that in WT controls (1 in 198) (Figure 1a, fourth panel). However, B7-H4 deletion did not affect the development of T-cell acute lymphoblastic leukemia induced by activated Notch1 during the serial transplantation (Supplementary Figures $2 \mathrm{a}-\mathrm{g}$ ). These results strongly indicate that $\mathrm{B} 7-\mathrm{H} 4$ may act as a tumor suppressor specifically for $\mathrm{AML}$ and does not affect functions of hematopoietic stem cells as we previously described, ${ }^{10}$ which suggests that $\mathrm{B} 7-\mathrm{H} 4$ may be an ideal target for the elimination of LICs. 


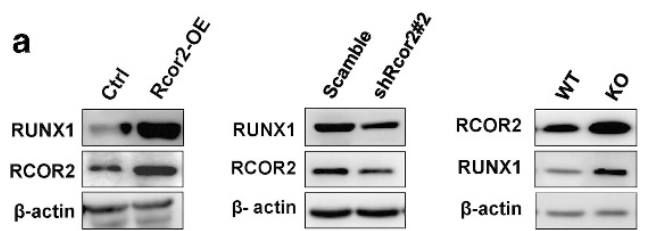

b

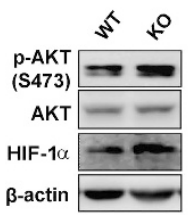

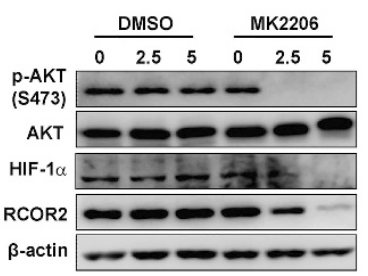

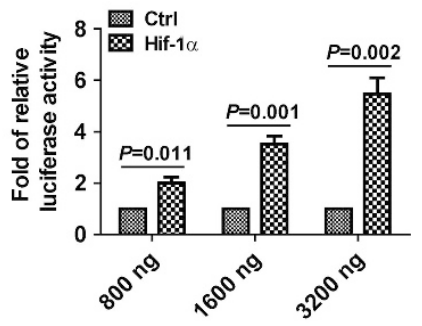

C

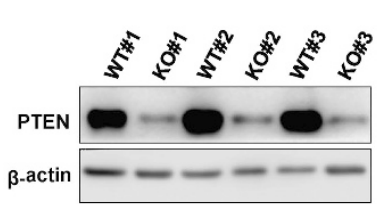

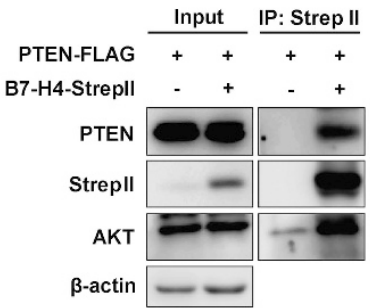

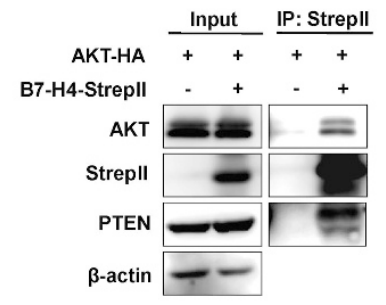

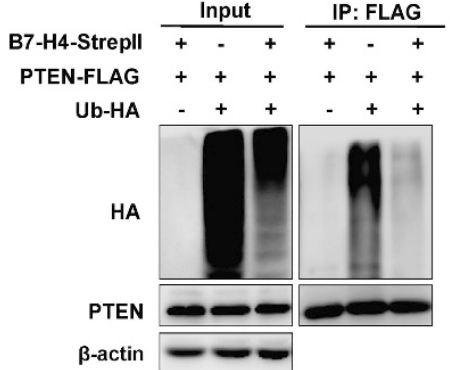

Figure 2. B7-H4 suppresses RCOR2 to reduce RUNX1 expression through PTEN/AKT signaling. (a) Protein levels of RUNX1 and RCOR2 were evaluated in Rcor2-over-expressing C1498 cells (first panel), Rcor2-knockdown C1498 cells (second panel) and WT and B7-H4-null BM leukemia cells (third panel) using immunoblotting. (b) Expression levels of p-AKT, AKT and HIF-1 $\alpha$ were measured in the WT and B7-H4-null BM AML cells (first panel). BM AML cells were treated with the AKT inhibitor MK2206 at the indicated doses $(0,2.5$ and $5 \mu \mathrm{m})$ for $72 \mathrm{~h}$ followed by the evaluation of the protein levels of p-AKT, AKT, HIF-1 $\alpha$ and RCOR2 using immunoblotting (second panel). Luciferase activity was measured in $293 \mathrm{~T}$ cells transfected with a luciferase reporter containing the Rcor2 promoter (-1770-719) and indicated doses of HIF-1 $\alpha$ plasmid, and normalized by Renilla. The relative luciferase activity of HIF- $1 \alpha$ were further standardized to that of control vector $(n=6$, third panel). (c) Protein levels of PTEN were measured in WT and B7-H4-null AML cells using immunoblotting (first panel). Strepll-tagged B7-H4 and Flag-tagged (a specific tag with eight amino acids for detection) PTEN were overexpressed in 293T cells, and their lysates were co-immunoprecipitated using strep-tactin sepharose followed by western blotting analysis for Flag (PTEN) and endogenous levels of AKT (second panel). Strepll-tagged B7-H4 and HAtagged AKT were overexpressed in 293T cells, and their lysates were co-immunoprecipitated (IP) using strep-tactin sepharose followed by western blotting analysis for HA (AKT) and endogenous levels of PTEN (third panel). Strepll-tagged B7-H4, Flag-tagged PTEN and HA-tagged ubiquitin were overexpressed in 293T cells followed by the addition of the proteasome inhibitor MG132 in culture during the last $4 \mathrm{~h}$. The lysates were immunoprecipitated using an anti-Flag M2 affinity gel followed by western blotting for HA (ubiquitin) (fourth panel).

To further elucidate the potential mechanisms leading to the enhanced proliferation of LICs, Wright-Giemsa staining was conducted and revealed that there was a much higher number of immature B7-H4-null blast cells in the bone marrow (BM) compared to that of the WT counterparts $(36.7 \pm 3.4$ vs $18.5 \pm 1.6 \%$, Supplementary Figure 3a), consistent with the increased infiltration of B7-H4-null leukemia cells in the spleen and liver as indicated by the changes of relative weights and hematoxylin/eosin staining (Supplementary Figures $3 \mathrm{~b}$ and $\mathrm{c}$ ). Consistently, the frequency of B7-H4-null Mac- $1^{+} \mathrm{Gr}-1^{-}$leukemia cells (Gr-1 expression level indicates the extent of myeloid cell differentiation) in the peripheral blood increased markedly to twofold greater than that in WT controls upon primary transplantation $(38.7 \pm 1.2 \%$ vs $17.2 \pm 3.4 \%$, Figure 1b). Consistent with in vivo data, B7-H4-null LICs had marked decreased ability to differentiate to $\mathrm{Mac}-1^{+} \mathrm{Gr}-1^{+}$mature leukemia cells in vitro upon stimulation by phorbol 12-myristate 13-acetate than WT counterparts (Supplementary Figure 3d). Moreover, the subsequent secondary and tertiary transplantations revealed similar changes in the differentiation status of B7-H4-null leukemia cells and displayed as a marked elevation in the percentage of the Mac- $1^{+} \mathrm{Gr}-1^{-}$cell population (Figure $1 \mathrm{~b}$ ).

To ask how B7-H4 influences the differentiation of LICs, we performed messenger RNA-sequencing analysis using WT and B7$\mathrm{H} 4-$ null Mac- $1^{+} \mathrm{C}-\mathrm{Kit}^{+} \mathrm{LICs}$. Interestingly, the mRNA-sequencing data revealed that several pathways other than immune regulation, including transcription (most markedly changed) and chromosome and chromatin organization, were also markedly altered (Supplementary Figure 4a). Several candidate genes related to transcription factors that may be important for the stemness of cancer stem cells (Rcor2, Klf2, Klf4, Klf6 and Hif-1a) and LIC differentiation (Cebpa, Gata2 and Pu.1), but not for self-renewal, were notably changed in B7-H4-null LICs (Supplementary Figures 4a and b). The expression levels of some potential targets (Rcor2, Klf4, Cebpa, Gata2, Runx1 and Hif-1a) were further determined using quantitative reverse transcription-polymerase chain reaction (Figure 1c, first panel and Supplementary Table 1), which showed a significant increase in B7-H4-null LICs than that in WT counterparts.

Rcor2 has been involved in multiple functions, including cell differentiation induction, self-renewal maintenance and epigenetic modifications in different cell types, ${ }^{11-13}$ which promotes us to test its potential connections with the phenotypes in B7-H4-null leukemic mice. Intriguingly, we revealed that the RCOR2 protein level was substantially elevated in B7-H4-null AML cells (Figure 1C, second panel). B7-H4-null AML cells were then treated with Rcor2-targeting short hairpin RNA (shRcor\#2, Supplementary Table 1) followed by the assessment of the in vivo engraftment. The mice transplanted with the Rcor2-knockdown B7-H4-null AML cells developed leukemia much more slowly than that of mice transplanted with B7-H4-null leukemia cells, which was comparable to their WT counterparts (Figure 1c, third panel and Supplementary Figure $4 \mathrm{~d}$ ). These results demonstrate that B7-H4 inhibits leukemogenesis via downregulation of RCOR2.

Because we also observed that RUNX1 was highly upregulated in B7-H4-null LICs (Figure 1C), which has been reported to maintain the stemness of both hematopoietic stem cells and LICs in a dosedependent manner, ${ }^{14,15}$ this promoted us to examine whether RCOR2 directly controls the expression of RUNX1 to inhibit the differentiation of B7-H4-null LICs. We thus ectopically expressed or knocked down Rcor2 in a mouse AML cell line (C1498) and observed that RUNX1 level was notably upregulated or reduced (Figure 2a, first to second panel). In addition, we further demonstrated that Rcor 2 could directly transactivate Runx 1 as 
evaluated by using a luciferase reporter (pGL3-Runx1, Supplementary Figure 5a) or a chromatin immunoprecipitation analysis (Supplementary Figure $5 \mathrm{~b}$ and Supplementary Table 1). Consistently, RUNX1 and RCOR2 protein levels were markedly upregulated in B7-H4-null AML cells than those of WT controls (Figure $2 \mathrm{a}$, third panel). To rule out the connections between B7-H4 and RCOR2, we examined several signaling pathways that might be regulated through $\mathrm{B} 7-\mathrm{H} 4$ and eventually observed that serine/ threonine kinase 1 (AKT) signaling was notably strengthened in B7H4-null AML cells (Figure 2b, first panel). Interestingly, RCOR2 level was indeed significantly reduced after treatment with an AKTspecific inhibitor (MK2206) for $72 \mathrm{~h}$ (Figure 2b, second panel). Furthermore, we revealed that HIF-1a could directly bind to the promoter of Rcor2 as determined by a luciferase reporter (Figure 2b, third panel). Consistently, both messenger RNA and protein levels of HIF-1a were remarkably upregulated in B7-H4-null AML cells (Figure $1 \mathrm{c}$ and Figure $2 \mathrm{~b}$, first panel). Inhibition of AKT signaling by MK2206 also reduced the HIF-1a and ROCR2 levels, indicating HIF-1a is one of the transcription factors involved in the AKTmediated ROCR2 activation (Figure 2b, second panel).

Because phosphatase and tensin homolog deleted on chromosome 10 (PTEN) is the major negative regulator of AKT phosphorylation, we also examined its levels in WT and B7-H4null AML cells, and showed that PTEN was markedly downregulated in B7-H4-null leukemia cells (Figure 5c, first panel). Considering that B7-H4 contains only two amino acids in the intracellular domain, ${ }^{7}$ we hypothesized that $\mathrm{B} 7-\mathrm{H} 4$ may be present in the cytoplasm or nucleus to regulate PTEN level. Indeed, B7-H4 existed in both the cytoplasm and nucleus as determined by immunoblotting (Supplementary Figure 5c). Immunofluorescence staining with either B7-H4-over-expressing C1498 cells or mouse AML cells also showed that B7-H4 could be detected on the cell membrane, in the cytoplasm or in the nucleus (Supplementary Figures $5 \mathrm{~d}$ and e). Interestingly, when $\mathrm{B} 7-\mathrm{H} 4$ was pulled down in PTEN-/B7-H4-over-expression 293T cells, both ectopically expressed PTEN and endogenous AKT could be easily detected in the immunoprecipitated cytoplasmic or nuclear samples, especially in cytoplasm (Figure 2c, second panel and Supplementary Figure 5f). Meanwhile, both ectopically expressed AKT and endogenous PTEN were clearly observed when B7-H4 was pulled down from AKT-/B7-H4-over-expressing 293T cells (Figure 2c, third panel). Furthermore, both overall level of ubiquitination and endogenous PTEN ubiquitination was markedly decreased following the overexpression of B7-H4 (Figure 2C, fourth panel), indicating that it has additional functions in the regulation of ubiquitination, although $\mathrm{B} 7-\mathrm{H} 4$ did not affect the transactivation of PTEN (Supplementary Figure $5 \mathrm{~g}$ ).

RCOR2 is usually considered a transcriptional repressor for the maintenance of the self-renewal of embryonic stem cells, neural stem cells. ${ }^{12}$ Surprisingly, our study showed that RCOR2 enhances the expression of RUNX1, indicating that this protein serves as a coactivator in LICs. Further studies are required to delineate how RCOR2 shifts between a transcriptional repressor and a coactivator and to determine how this protein controls the expression levels of RUNX1. Herein, we present the first evidence that B7-H4 acts as a tumor suppressor to through PTEN/AKT/HIF-1a/RCOR2/RUNX1 pathways to promote the differentiation of LICs, but does not impair the normal functions of hematopoietic stem cells (Supplementary Figure 5h). B7-H4 expression level is positively correlated with the overall survival of AML patients. Activation of B7-H4 by functional antibodies or other small molecule chemicals may be an efficient way to eliminate LICs. These results also indicate targeting LICs through its downstream molecules, such as RCOR2, may be other valuable strategies for the treatment in leukemia.

\section{CONFLICT OF INTEREST}

The authors declare no conflict of interest.

\section{ACKNOWLEDGEMENTS}

We appreciate $\mathrm{Dr}$ Junfa $\mathrm{Xu}$ at Guangdong Medical University, Guangdong Dongguan, for his kindness in providing us the B7-H4 complementary DNA. This work was supported by grants from National Natural Science Foundation of China (81370654, 81422001, 81570093, 81471524 and 81600614), the 1000-Youth Elite Program, National Basic Research Program of China (973Program, 2014CB965000; 2015CB910403), and Natural Science Foundation of Shanghai (17ZR1415500).

\section{AUTHOR CONTRIBUTIONS}

$F X, Y Z, L X, H J, Y L, Z Y$ and $J Z$ designed the experiments, performed the experiments, analyzed data and wrote the paper; $H Z, C C, L L, X H, X H, X F, X L, F Z$, HG, JW and YC performed the experiments; and CCZ and G-QC provided reagents and helped with the experiments.

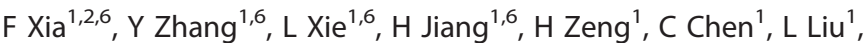

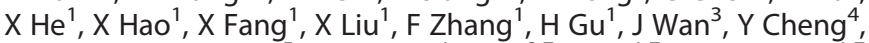
CC Zhang ${ }^{5}$, G-Q Chen ${ }^{1}, \mathrm{Y} \mathrm{Lu}{ }^{2,7}, \mathrm{Z} \mathrm{Yu}^{1,7}$ and J Zheng ${ }^{1,7}$

${ }^{1}$ Department of Pathophysiology, Hongqiao International Institute of Medicine, Shanghai Tongren Hospital/Faculty of Basic Medicine, Key Laboratory of Cell Differentiation and Apoptosis of Chinese Ministry of Education, Shanghai Jiao Tong University School of Medicine, Shanghai, China;

${ }^{2}$ Institute and Department of Endocrinology and Metabolism, Shanghai Ninth People's Hospital, Shanghai Jiao Tong University School of Medicine, Shanghai, China:

${ }^{3}$ Department of Hematology, Xinhua Hospital, Shanghai Jiao Tong University School of Medicine, Shanghai, China;

${ }^{4}$ Department of Hematology, Shanghai Ninth People's Hospital, Shanghai Jiao Tong University School of Medicine, Shanghai, China and ${ }^{5}$ Departments of Physiology and Developmental Biology, UT Southwestern Medical Center, Dallas, TX, USA

E-mail: zhengjunke@shsmu.edu.cn or yuzhuo78@aliyun.com or luyingli2008@126.com

${ }^{6}$ These authors contribute equally to this work.

${ }^{7}$ These authors share senior authorship.

\section{REFERENCES}

1 Jordan CT. Unique molecular and cellular features of acute myelogenous leukemia stem cells. Leukemia 2002; 16: 559-562.

2 Zheng J, Umikawa M, Cui C, Li J, Chen X, Zhang C et al. Inhibitory receptors bind ANGPTLs and support blood stem cells and leukaemia development. Nature 2012; 485: 656-660.

3 Tettamanti S, Biondi A, Biagi E, Bonnet D. CD123 AML targeting by chimeric antigen receptors: a novel magic bullet for AML therapeutics? Oncoimmunology 2014; 3: e28835.

4 Majeti R, Chao MP, Alizadeh AA, Pang WW, Jaiswal S, Gibbs KD Jr et al. CD47 is an adverse prognostic factor and therapeutic antibody target on human acute myeloid leukemia stem cells. Cell 2009; 138: 286-299.

5 Iwasaki M, Liedtke M, Gentles AJ, Cleary ML. CD93 marks a non-quiescent human leukemia stem cell population and is required for development of MLLrearranged acute myeloid leukemia. Cell Stem Cell 2015; 17: 412-421.

6 Zhang F, Liu X, Chen C, Zhu J, Yu Z, Xie J et al. CD244 maintains the proliferation ability of leukemia initiating cells through SHP-2/p27kip1 signaling. Haematologica 2017; 102: 707-718.

7 Prasad DV, Richards S, Mai XM, Dong C. B7S1, a novel B7 family member that negatively regulates $T$ cell activation. Immunity 2003; 18: 863-873.

8 Dong Q, Ma X. B7-H4 expression is associated with tumor progression and prognosis in patients with osteosarcoma. BioMed Res Int 2015; 2015: 156432.

9 Rahbar R, Lin A, Ghazarian M, Yau HL, Paramathas S, Lang PA et al. B7-H4 expression by nonhematopoietic cells in the tumor microenvironment promotes antitumor immunity. Cancer Immunol Res 2015; 3: 184-195.

10 Zheng J, Umikawa M, Zhang S, Huynh $\mathrm{H}$, Silvany R, Chen BP et al. Ex vivo expanded hematopoietic stem cells overcome the MHC barrier in allogeneic transplantation. Cell Stem Cell 2011; 9: 119-130.

11 Qureshi IA, Gokhan S, Mehler MF. REST and CoREST are transcriptional and epigenetic regulators of seminal neural fate decisions. Cell Cycle 2010; 9: 4477-4486.

12 Yang P, Wang Y, Chen J, Li H, Kang L, Zhang $Y$ et al. RCOR2 is a subunit of the LSD1 complex that regulates ESC property and substitutes for 
SOX2 in reprogramming somatic cells to pluripotency. Stem Cells 2011; 29: 791-801.

13 Saleque S, Kim J, Rooke HM, Orkin SH. Epigenetic regulation of hematopoietic differentiation by Gfi- 1 and Gfi-1b is mediated by the cofactors CoREST and LSD1. Mol Cell 2007; 27: 562-572.

14 Fu L, Fu H, Tian L, Xu K, Hu K, Wang J et al. High expression of RUNX1 is associated with poorer outcomes in cytogenetically normal acute myeloid leukemia. Oncotarget 2016; 7: 15828-15839.

15 Tober J, Yzaguirre AD, Piwarzyk E, Speck NA. Distinct temporal requirements for Runx1 in hematopoietic progenitors and stem cells. Development 2013; 140: 3765-3776.

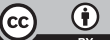

This work is licensed under a Creative Commons Attribution 4.0 International License. The images or other third party material in this article are included in the article's Creative Commons license, unless indicated otherwise in the credit line; if the material is not included under the Creative Commons license, users will need to obtain permission from the license holder to reproduce the material. To view a copy of this license, visit http://creativecommons.org/licenses/ by/4.0/

(c) The Author(s) 2017

Supplementary Information accompanies this paper on the Leukemia website (http://www.nature.com/leu)

\section{NK cell dynamics and association with molecular response in early chronic phase chronic myelogenous leukemia (CML-CP) patients treated with nilotinib}

Leukemia (2017) 31, 2264-2267; doi:10.1038/leu.2017.235

The main treatment goal in chronic myelogenous leukemia (CML) is the achievement of deep molecular response (DMR), which makes disease progression highly unlikely and may even trigger discontinuation of tyrosine kinase inhibitor (TKI) therapy. Second generation TKIs (for example, nilotinib, dasatinib) induce DMR with a higher probability compared to imatinib. ${ }^{1,2}$ In recent years immunological factors are increasingly acknowledged as

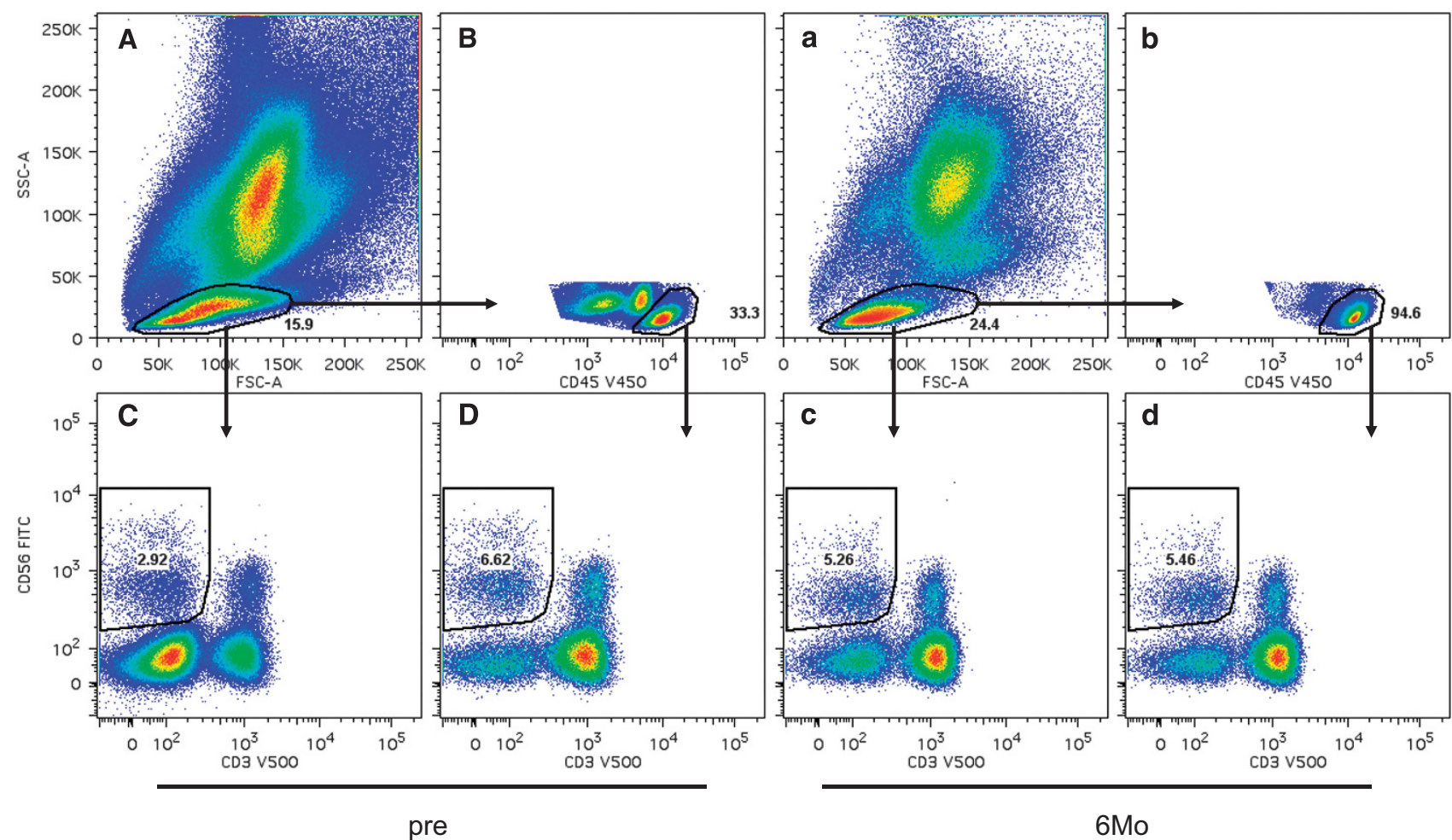

Figure 1. Gating strategy to define NK cells numbers. A representative flow cytometry analysis of whole blood from a CML patient before (upper case letters) and at month 6 (lower case letters) of nilotinib therapy is given. Lymphocytes were gated using FSC and SSC (A, a). To exclude CD45 low leukocytes, such as basophils and hematopoietic progenitors (see also Supplementary Figure 1), lymphocytes were additionally defined by CD45 $5^{\text {high }}$ expression $(\mathbf{B}, \mathbf{b})$. The proportion of $C D 56^{+} C D 3^{\text {neg }}$ NK cells is shown among leukocytes gated based solely on scatter characteristics $(\mathbf{C}, \mathbf{c})$ or as percentage of $C D 45^{\text {high }}$ lymphocytes $(\mathbf{D}, \mathbf{d})$. In case the lymphocyte gate contains a large proportion of nonlymphocytic leukocytes or hematopietic progenitor cells in therapy naïve CML patients (C), the percentage of NK cells is disproportionately low, when compared to the percentage of NK cells gated on CD45 high lymphocytes (D). This difference (compare $c$ and d) is negligible in the same patient during nilotinib therapy, as the TKI therapy depleted contaminating clonal cells in the lymphocyte gate. 